\title{
ACONSELHAMENTO PÓS-TESTE ANTI-HIV: ANÁLISE À LUZ DE UMA TEORIA HUMANÍSTICA DE ENFERMAGEM
}

Anti-HIV After-Test counselling:

Analysis at a light of a Nursing humanistic theory

Consejería Pós Test Anti-HIV:

Análisis a luz de una teoría humanistica de enfermería

Maria Alix Leite Araújo

Francisca Lucélia Ribeiro de Farias

Alanna Virgínia Brito Rodrigues

\begin{abstract}
Resumo
Este trabalho analisa o aconselhamento pós-teste anti-HIV em Unidades Básicas de Saúde. Trata-se de um estudo qualitativo desenvolvido em Unidades Básicas de Saúde da Família de Fortaleza (UBASF). 0 trabalho de campo ocorreu pela observação do atendimento de 12 enfermeiros em consultas de pré-natal. 0 referencial teórico de análise foi a Teoria Humanística de Enfermagem de Paterson e Zderad visto que a Enfermagem, segundo a teoria, implica um tipo especial de encontro entre seres humanos. A análise baseou-se no conceito de diálogo, tendo como variáveis seus elementos estruturais: o encontro, o relacionamento, a presença e o chamado, e a resposta. Observou-se que a assistência às gestantes não atingiu o relacionamento EU-TU, ou seja, o relacionamento sujeito-sujeito, com a presença do diálogo genuíno. Prevaleceu o relacionamento EU-ISSO, sujeito-objeto. As consultas eram rápidas e puramente mecânicas, levando esses profissionais, em certos momentos, a fugirem de uma assistência humanizada.
\end{abstract}

Palavras-chave: Aconselhamento. Teoria de Enfermagem. Atenção Primária à Saúde.

\begin{abstract}
This work analyzes the counselling after anti-HIV test in Basic Units of Health. It is a qualitative study developed in Family's Basic Units of Health in the city of Fortaleza (UBASF). The field work happened by the observation of the 12 male nurses attendance in consultations of prenatal. The theoretical referencial of analysis was the Paterson \& Zderad's Humanistic Theory of Nursing, seen that in Nursing, according to the theory, implicates in a special type of encounter among human beings. The analysis was based on the concept of dialogue, having as variables its structural elements: the encounter, the relationship, the presence and the call and the answer. It was observed that the assistance to the pregnant didn't reach the relationship I-YOU, in other words, the relationship subject-subject, with the presence of the authentic dialogue. The relationship that prevailed was the I-THAT, subject-object. The consultations were fast and purely mechanics, leading those professionals in certain moments to escape from a humanized assistance.
\end{abstract}

\section{Resumen}

Este trabajo analiza el pos test de consejería anti-HIV en las Unidades Básicas de Salud. Es un estudio cualitativo desarrollado en las Unidades Básicas de Salud de la Familia en la ciudad de Fortaleza (UBASF). El trabajo de campo sucedió por la observación de la asistencia de 12 enfermeros en las consultas de pré-natal. El referencial teórico de análisis fué la Teoría Humanistica de Paterson y Zderad de Enfermería, visto en la enfermería, según la teoría, implica en un tipo especial de encuentro entre los seres humanos. El análisis era basado en el concepto de diálogo, teniendo como las variables sus elementos estructurales: el encuentro, la relación, la presencia y el llamamiento y la respuesta. Fue observado que la ayuda a la embarazada no alcanzó la relación YOUSTED, en otros términos, el sujeto-sujeto de la relación, con la presencia del diálogo auténtico. La relación que prevaleció era el YO-AQUEL, sujeto-objeto. Las consultas eran rápidas y puramente mecánicas, llevando a esos profesionales en ciertos momentos a no brindar de una ayuda humanizada. 


\section{INTRODUÇÃO}

A Síndrome da Imunodeficiência Adquirida (AIDS), denominada como a mais recente pandemia da humanidade, constitui-se hoje um dos mais sérios problemas mundiais de saúde pública. É uma doença crônica causada pelo Vírus da Imunodeficiência Humana (HIV), ocasionando perda progressiva da imunidade celular e, conseqüentemente, o aparecimento de infecções oportunistas ${ }^{1}$.

Ao longo dos anos, a AIDS passou por mudanças no perfil epidemiológico em que prevaleceram diferentes populações. Hoje existem no mundo cerca de 23 milhões de pessoas infectadas pelo HIV, das quais aproximadamente $40 \%$ são mulheres, caracterizando o que foi denominado de feminização da epidemia. Como conseqüência, ocorreu um aumento de casos de AIDS em crianças, ocasionado especialmente pela transmissão vertical (TV), ou seja, a transmissão do vírus para o bebê durante a gestação, o parto ou aleitamento materno².

0 aumento da TV do HIV apresenta-se como um grande desafio para a saúde pública, pois esta modalidade de transmissão é a principal via de infecção do HIV na população infantil. 0 primeiro caso notificado no Brasil foi em 1985 e, atualmente, cerca de 85\% dos casos de AIDS em crianças menores de 13 anos ocorreram por essa forma de transmissão?

Com o objetivo de reduzir as taxas de transmissão vertical do HIV, o Brasil adotou a como política pública 0 oferecimento do teste anti-HIV a todas as gestantes durante 0 acompanhamento pré-natal ${ }^{3}$. 0 tratamento da gestante com a quimioprofilaxia reduz drasticamente o risco de transmissão do HIV para 0 bebê $\hat{A}^{4-5}$, o que justifica o oferecimento do teste por ocasião dos atendimentos nos serviços de atenção a saúde da mulher, especialmente no pré-natal.

0 Ministério da Saúde normatizou na rotina do atendimento pré-natal o oferecimento do exame antiHIV, com o consentimento verbal da gestante, tendo como requisito básico sessões de aconselhamento pré e pós-teste. Este se pauta especialmente em um processo de escuta às demandas, troca de informações e apoio emocional ${ }^{6}$.

0 aconselhamento no pré-natal configura-se em um diálogo que visa a estabelecer uma relação de confiança entre os interlocutores e a oferecer à gestante condições para que avalie sua condição de vulnerabilidade e riscos pessoais de portar o HIV, tome decisões e encontre maneiras realistas, ou seja, maneiras viáveis de enfrentar seus problemas relacionados às DST/HIV/AIDS ${ }^{6}$. Um estudo aponta que, para pacientes com DST, o aconselhamento melhora os índices de adesão ao tratamento, o retorno para receber o resultado dos exames, o tratamento do parceiro sexual e uso do preservativo durante o tratamento?.

A detecção precoce do HIV em gestantes deve acontecer especialmente nas Unidades Básicas de Saúde da Família (UBASF), em razão da facilidade de acesso geográfico da população e por estas unidades serem responsáveis em garantir boas coberturas de pré-natal. Por esse motivo, elas devem se tornar fortes aliadas na prevenção vertical do HIV, considerando ser esta população prioritária para a Estratégia de Saúde da Família.

A solicitação e realização do teste anti-HIV na gestação favorece a identificação de portadoras do vírus e a possibilidade de encaminhamento precoce para tratamento e acompanhamento adequados da gestante. 0 acompanhamento pré-natal adequado de uma gestante soropositiva inicia-se com o aconselhamento pós-teste e o apoio emocional para o enfrentamento do resultado para consigo mesmo e junto à família.

A inserção do aconselhamento nas UBASF constituise um grande desafio para gestores e profissionais de saúde, pois necessita de muitos mecanismos de superação para que resultados satisfatórios sejam obtidos. Um estudo sobre a adesão das gestantes ao exame anti-HIV mostrou que ainda é muito baixo 0 percentual de testagem no pré-natal, indicando que existem limitações para a realização do aconselhamento e testagem na atenção básica ${ }^{8}$.

Um dos aspectos que pode contribuir para uma boa cobertura de testagem do HIV no pré-natal é a realização de um aconselhamento de qualidade. Essa qualidade pode ser traduzida especialmente na escuta às demandas das usuárias, no apoio emocional para ajudálas a enfrentar os conflitos que normalmente aparecem por ocasião da testagem e na troca de informações. Os profissionais mesmo depois de treinados priorizam o conteúdo informativo e os aspectos cognitivos do aprendizado, em detrimento ao componente emocional ${ }^{9}$, o que pode fazer com que muitas mulheres deixem de fazer o exame com medo do resultado ${ }^{10}$.

Desta forma, visando a contribuir para a melhoria da assistência profissional às gestantes no pré-natal, buscamos desenvolver esta pesquisa, com o objetivo de conhecer como os profissionais de enfermagem desenvolvem o processo de aconselhamento pós-teste anti-HIV para mulheres grávidas, analisando 0 aconselhamento à Luz da Teoria Humanística de Paterson e Zderad. Essa teoria tem uma relação estreita com o aconselhamento, quando valoriza a relação entre o profissional e o paciente e as questões subjetivas presentes nessa relação. 


\subsection{A Teoria Humanística de Paterson e Zderad}

A teoria humanística de Paterson e Zderad teve origem no existencialismo, com influência da fenomenologia, e tem como pressuposto que a pessoa deve tomar decisões, dar direção e sentido à sua própria vida. Enfatiza a vida como é vivida e, com base nesta visão, sugere três conceitos que são a base da Enfermagem humanística: o diálogo, a comunhão e a Enfermagem fenomenológica ${ }^{11}$.

Baseia-se no diálogo, que é considerado um colóquio, uma conversação entre duas ou mais pessoas. Para a referida teoria, o diálogo é mais que uma conversação, é um relacionamento em que ocorre um verdadeiro partilhar, uma relação intersubjetiva, isto é, a relação de um indivíduo único (EU) com o outro também único (TU) ${ }^{11}$.

A Enfermagem é entendida como um diálogo vivo, que objetiva a busca do bem-estar físico e psicológico do ser ${ }^{11}$. Durante 0 aconselhamento pós-teste, para que este objetivo seja atingido, faz-se necessário que os profissionais estejam conscientes que trazem consigo valores, preconceitos, mitos e expectativas. Estes elementos da teoria estão envolvidos com a experiência intersubjetiva que envolve 0 aconselhamento e incorporam os elementos de estrutura da teoria que são: o encontro, o relacionamento, a presença e 0 chamado e resposta.

Para 0 aconselhamento, suas bases conceituais têm raízes em teorias da psicologia, mais especificamente na teoria centrada na pessoa, que a reconhece como um sujeito autônomo, capaz de tomar decisões ${ }^{12-13}$.

0 encontro é caracterizado pelo agrupamento de duas ou mais pessoas que trazem consigo expectativas que interferem na relação ${ }^{11}$. Porém, para a teoria, o encontro só é iniciado quando se atinge a singularidade dos indivíduos, levando em consideração os aspectos subjetivos presentes tanto no profissional como na usuária. Portanto, não significa somente estar presente fisicamente, mas criar um ambiente onde realmente as pessoas se sintam acolhidas ${ }^{14}$, considerando as suas demandas de maneira diferenciada e singular.

As usuárias e enfermeiros devem ser vistos como seres humanos singulares, que interagem consigo mesmo, com os outros e com o ambiente, e que apresentam visões de mundo influenciadas pelas suas diferenças sociais e culturais.

0 relacionamento é estar com o outro e é precedido pelo encontro. Segundo a teoria, existem duas formas de relacionamento: o EU-TU e EU-ISSO. Para que a relação se dê como EU-TU, devem existir trocas, em que ambos saem transformados da relação. Estas trocas devem atingir a autenticidade e singularidade do ser. $\mathrm{Na}$ relação EU-ISSO, o enfermeiro vê o cliente como um objeto, sem condições de tomar decisões e sem autonomia ${ }^{11}$.

Uma relação dialógica exige que os indivíduos estejam presentes em sua totalidade. Está além do desenvolvimento das habilidades técnicas, pois compreende um "estar com", que envolve a habilidade de proporcionar um ambiente favorável para que a usuária também esteja presente em sua totalidade. Para tanto, precisam tratar as usuárias como pessoas autônomas e capazes de tomar decisões.

Ainda segundo a teoria, a presença é a qualidade de estar aberto um para o outro, disponível para alcançar a relação EU-TU. É elemento fundamental e necessário para se alcançar um bom relacionamento. 0 chamado e a resposta podem acontecer de forma verbal e não verbal e referem-se à capacidade dos profissionais de enfermagem de se relacionarem com os aspectos subjetivos, como o medo e a insegurança, e os aspectos objetivos, como dor, febre, dentre outros ${ }^{11}$.

Para a realização do aconselhamento pós-teste antiHIV, esses elementos envolvidos na teoria humanística de Paterson e Zderad devem estar presentes, uma vez que a realização deste teste, especialmente quando existe percepção de risco na usuária, se caracteriza como um momento delicado, que normalmente está acompanhado de tensão e medo. Daí a importância de o profissional de saúde estar devidamente treinado para promover um efetivo encontro, proporcionando um ambiente de acolhimento, de escuta e de entendimento das emoções, que podem estar presentes de forma diferenciada para cada gestante.

0 aconselhamento para DST/HIV/AIDS tem, portanto, uma relação estreita com a teoria humanística de Paterson e Zderad, pois ambos pautam seus conceitos fundamentais na relação e no diálogo entre os envolvidos. A prática do aconselhamento é fundamental para a adesão ao teste e ao diagnóstico do HIV e tem três componentes básicos: apoio educativo, apoio emocional e avaliação de riscos, que exigem preparação da equipe para acolher a subjetividade dos usuários.

\section{METODOLOGIA}

Trata-se de uma pesquisa de cunho descritivo e de natureza qualitativa, por se adequar ao estudo de significados, motivos, aspirações e crenças, valores e atitudes, o que corresponde a um espaço mais profundo das relações humanas ${ }^{15}$. A pesquisa qualitativa permite compreender o problema no meio em que ele ocorre, sem criar situações artificiais que mascaram a realidade, ou que levam a interpretações ou generalizações equivocadas ${ }^{16}$. 
0 trabalho de campo aconteceu em três Unidades Básicas de Saúde da Família (UBASF), localizadas na região administrativa da Secretaria Executiva Regional IV (SER IV), na cidade de Fortaleza-Ceará. Estas UBASF têm um total de 12 profissionais de enfermagem, 12 médicos, 4 cirurgiões dentistas, 12 auxiliares de enfermagem e 60 agentes comunitários de saúde (ACS), todos divididos em 12 equipes do Programa da Saúde da Família (PSF). Estas equipes atendem, em média, 12.000 famílias $^{17}$.

$\mathrm{Na}$ cidade de Fortaleza em 2001, foram cadastrados 6.408 gestantes, das quais 6.147 foram acompanhadas com consultas de pré-natal, correspondendo a um percentual de $94,7 \%$. Na SER IV onde estão inseridas as referidas UBASF pesquisadas, foram acompanhadas $93,3 \%$ das gestantes cadastradas, um índice bastante elevado e que chegou muito próximo da meta de cobertura preconizada pelo Ministério da Saúde, que é de $100 \%$. No mesmo ano, foram realizadas 13.804 visitas domiciliares, na proporção de um enfermeiro para cada 1.150 visitas $^{17}$.

Estas UBASF foram selecionadas para participar da pesquisa devido à sua boa cobertura de pré-natal em gestantes (em torno de $90 \%$ ) e ao fácil acesso de uma das pesquisadoras, que já estagiou em uma das UBASF, o que favoreceu a sua interação com os profissionais de saúde.

A modalidade de coleta dos dados foi a observação não participante. Foram observados os atendimentos de 12 enfermeiros, totalizando 24 consultas. 0 trabalho de campo foi realizado no turno da manhã, pois 0 atendimento ocorre nesse turno. As enfermeiras que participaram do estudo deveriam realizar atendimento pré-natal, e a observação restringiu-se a gestantes que já haviam realizado o teste anti-HIV e estavam aguardando o resultado. 0 objetivo era assistir à entrega do resultado do teste anti-HIV juntamente com 0 aconselhamento pós-teste. As gestantes eram abordadas antes do atendimento para se certificar de que haviam colhido o sangue e, portanto, realizado 0 teste. Nesse momento, era também solicitada autorização para participar do atendimento.

0 aconselhamento pós-teste representa 0 momento de entrega do exame anti-HIV e é muito mais carregado de tensão e ansiedade que a solicitação do teste (pré-teste), pois representa o momento real de descoberta ou não da soropositividade. Normalmente é a ocasião em que a pessoa reflete acerca dos riscos vivenciados e da possibilidade de portar o HIV.

Um roteiro de observação do tipo check list foi adaptado a partir do Manual de Aconselhamento elaborado pelo Ministério da Saúde para acompanhamento das consultas de entrega do resultado do teste anti-HIV ${ }^{18}$. Foram utilizados do manual os itens que estariam mais proximamente relacionados aos preceitos da teoria e relacionados ao estabelecimento do diálogo e do encontro, como por exemplo: A enfermeira identificou com clareza a demanda da gestante? Prestou apoio emocional? Utilizou linguagem compatível com a cultura da gestante? Facilitou à gestante a expressão dos sentimentos?

As observações de campo foram realizadas com 12 enfermeiros e 12 gestantes. As enfermeiras que participaram do estudo deveriam fazer atendimento prénatal e entrega do teste anti-HIV. As gestantes já deveriam ter realizado o teste anti-HIV e estar aguardando o resultado do exame. Para tanto deveriam estar pelo menos na segunda consulta de pré-natal.

Os dados eram anotados no diário de campo e foram analisados a partir de leituras sucessivas do material coletado, confrontando com os dados observados e marcados no check list. As categorias foram definidas previamente a partir dos próprios elementos estruturais da teoria, que são o diálogo e 0 encontro. Estes elementos não podem ser analisados isolados dos outros componentes que são: 0 relacionamento, a presença e o chamado, e a resposta.

Visando a evitar qualquer constrangimento no momento das observações do atendimento, a entrevistadora fazia as anotações e marcava o check list somente imediatamente após os atendimentos.

0 estudo recebeu aprovação do comitê de ética e pesquisa da Universidade de Fortaleza (UNIFOR), e foram observados todos os preceitos éticos e as diretrizes e normas regulamentadoras de pesquisa envolvendo seres humanos da Resolução 196, de 10/10/1996. Foram garantidos 0 anonimato e o sigilo das informações.

A atividade foi também previamente autorizada pela diretora responsável pelas Unidades Básicas de Saúde da SER IV, bem como pelas enfermeiras que realizavam as consultas de pré-natal e as gestantes que participaram das consultas. Estas assinaram o termo de consentimento e foram esclarecidas quanto ao objetivo da pesquisa e período de coleta de dados. Tiveram assegurados o sigilo, 0 acesso aos dados e 0 direito de se retirarem do estudo a qualquer momento se acharem pertinente sem qualquer prejuízo.

\section{ANÁLISE E INTERPRETAÇÃO DOS DADOS}

Como citado anteriormente, as categorias foram definidas previamente a partir dos próprios elementos estruturais da teoria. Portanto, o diálogo e o encontro foram definidos como categorias gerais e incorporam 0 relacionamento, a presença, o chamado e a resposta, componentes essenciais também da estrutura da teoria. 


\subsection{Diálogo}

Para a realização do aconselhamento, o diálogo é fundamental. Este diálogo só se estabelece em um ambiente acolhedor e com espaço físico apropriado, para que questões de foro íntimo que geralmente estão envolvidos nesse processo possam ser discutidas. É preciso que se garantam a manutenção do sigilo, a privacidade das informações e uma adequada abordagem acerca das situações de foro íntimo. Na unidade, as salas são separadas por divisórias que não impedem que outros profissionais da unidade escutem a conversa; isso sem considerar as constantes interferências realizadas durante a consulta, que atrapalham a concentração dos envolvidos.

Estudos que analisaram o conceito de ambiente nesta referida teoria mostraram que o espaço físico pode não se apresentar como um aspecto de muita relevância, considerando que 0 ato de "vir a ser" pode ocorrer tanto em ambiente privativo como coletivo. 0 ambiente por si só não é impeditivo para que se estabeleça uma relação interativa, uma verdadeira comunhão entre a enfermeira e o cliente. Representa o desenvolvimento de uma atmosfera facilitadora, onde a ênfase está na qualidade do encontro ${ }^{14}$.

Apesar de 0 ambiente não representar somente 0 espaço físico, queremos salientar a importância deste espaço para que esta atmosfera se estabeleça. Interrupções constantes e falta de privacidade com certeza vão impedir um ambiente de permissividade, para que se possam elucidar questões do comportamento sexual, o uso de drogas, uso ou não de preservativos, enfim, aspectos da avaliação de riscos para o HIV, necessários e recomendados quando da solicitação e entrega do teste anti-HIV.

Outro problema identificado nas observações do trabalho de campo foi o quantitativo de atendimentos realizado diariamente pelos profissionais enfermeiros, o que acarretou falta de tempo para se abrir ao diálogo e estarem inteiros na relação, o que com certeza exige maior disponibilidade pessoal para desenvolver a escuta.

A forma de organização interna do trabalho na unidade, que privilegiava a quantidade de atendimentos em detrimento da qualidade, exigiu do enfermeiro a realização de um grande volume de atendimentos muitas vezes em intervalos de tempo curtos, visando a alcançar as metas e garantir a produtividade, uma das formas de remuneração e complementação salarial. Essa situação faz com que se torne praticamente impossível o desenvolvimento de uma atmosfera que privilegie o diálogo. Por outro lado, na prática, a carga horária que o profissional de enfermagem cumpre é de seis horas, tornando-se desproporcional para o elevado número de usuários que necessitam de atendimento.

Vale ressaltar que estes profissionais trabalham com um sistema informatizado, com horário previsto para funcionamento, o que ocasiona uma certa limitação para a realização de cuidados a um grande número de clientes. Nas UBASF, pode-se observar a dificuldade enfrentada pelo profissional para realizar um atendimento que contemple minimamente os componentes da teoria. 0 grande volume de atendimentos os leva a realizá-los em um intervalo de tempo muito curto, o que na grande maioria das vezes não favorece uma concentração e interesse aprofundado pelas demandas da gestante.

Em outros momentos do atendimento ocorreram várias interrupções por colegas da própria instituição ou por outros clientes em busca de informações, situação que impedia claramente que houvesse 0 encontro e o chamado e resposta, condições propostas para que ocorra um atendimento humanizado.

\subsection{Encontro}

Foi observado que as mães apresentavam muitas expectativas em relação à consulta, muitas apresentavam ansiedade e aflição em relação ao resultados dos exames, principalmente 0 anti-HIV, e em relação à saúde da criança. Outras tinham uma expectativa maior com relação ao início da consulta, que demonstravam através da inquietação e tom de voz elevados.

Os profissionais referiram que suas expectativas eram as mesmas para todas as consultas e estavam muito mais voltadas para o atendimento clínico da gestante, como das queixas mais comuns, como azia, náuseas e outros sintomas comuns neste período. Não passavam, portanto, de consultas de rotina. A princípio não pensam na possibilidade de um resultado positivo para HIV nas gestantes, o que os leva a vivenciar muita dificuldade na entrega do mesmo.

0 encontro verdadeiro dos enfermeiros com as gestantes das UBASF deve estar voltado não somente para os problemas e suas soluções, mas também para a comunhão e a partilha. Segundo a teoria humanística, 0 encontro precede o relacionamento, porém ambos só conseguem atingir seus objetivos quando são realizados conjuntamente. Este relacionamento pode ser feito de duas formas, dependendo de como a relação se estabeleça: EU-TU e EU-ISSO ${ }^{11-19}$, que se diferenciam em suas concepções filosóficas.

0 cuidado é um dos aspectos envolvidos no relacionamento entre o profissional de enfermagem e a usuária, e, durante o encontro, ambos devem se sentir valorizados. Para uma relação dialógica, faz-se necessária 
uma presença genuína, vivenciada na sua totalidade, tanto por parte do profissional como da usuária.

Nas unidades básicas, o relacionamento tornar-seá significante quando as enfermeiras deixarem de se prender a protocolos estabelecidos e inflexíveis e fizerem com que sua presença seja vista pela cliente como um mediador do cuidado, de alguém que está preocupado e interessado em ajudá-la, e com que, através desta relação, exista troca de experiências e empatia. Observa-se que a relação EU-ISSO é freqüente nas consultas de pré-natal nas UBASF. As orientações são repassadas através de um volume considerável de procedimentos normativos que deveriam ser adotados pela gestante sem levar em conta minimamente as condições e contextos de vida das mesmas.

Essas mulheres passam a ser consideradas como objetos quando são reduzidas a casos clínicos, a receptáculos de procedimentos técnicos sem capacidade de tomar decisões favoráveis à sua saúde. Além do mais, usam-se muitos termos técnicos, muitas vezes de difícil entendimento, considerando que essas mulheres na sua grande maioria têm pouco ou nenhum estudo.

$\mathrm{Na}$ maioria das vezes, as consultas resumiam-se a um procedimento mecânico, através do preenchimento do cartão da gestante com dados solicitados pelo sistema computadorizado, levantamento de questões referentes às queixas clínicas e exame obstétrico limitado à medição do fundo de útero. Poucas enfermeiras mostraram-se interessadas em ouvir além das queixas clínicas das gestantes, e estavam atentas a outras demandas verbais ou não verbais expressas, na tentativa de realizar um atendimento mais genuíno.

Ao desacreditarem a capacidade da gestante em resolver seus problemas, as enfermeiras a transformam em objeto. Sendo assim, o ser cuidado, no caso a gestante, passa a ser visto apenas como executor de procedimentos determinados pela profissional, impedindo, dessa forma, que o encontro e o diálogo realmente se estabeleçam, uma vez que se cria uma barreira entre o ser cuidado e o cuidador.

A clientela das UBASF é formada na sua grande maioria por pessoas pobres de pouca instrução e que acabam submetendo-se sem questionamentos a tudo o que o profissional fala e recomenda. Isso não significa necessariamente que entenderam as orientações ou que realizarão os procedimentos recomendados.

Portanto, cabe ao profissional quebrar esta barreira, tentando alcançar a relação EU-TU, dando maior abertura e estimulando, através de técnicas de comunicação, que a gestante deixe a timidez, o medo de falar e desabafar suas dúvidas e angústias. 0 ser cuidado será capaz de ver o profissional como sujeito se na relação existir o diálogo que valorize suas queixas e dê a ele a oportunidade de expressar suas angústias e solicitar esclarecimentos.

Nas unidades básicas de saúde, o chamado das gestantes pode acontecer através de um pedido de ajuda relacionado a um sangramento, a uma dor, dentre outros. Como resposta, o profissional de enfermagem executará os procedimentos que possam reduzir os desconfortos apresentados pela gestante. Este exemplo caracteriza a objetividade de ambos os seres.

Como chamado e resposta, podemos citar a presença das gestantes nas consultas de pré-natal, denotando a necessidade de conforto e segurança durante a gravidez, período de certa forma delicado na vida da mulher. Portanto, a Enfermagem é uma resposta aos cuidados e a um chamado de ajuda que se dá por meio da compreensão do significado de experiência de vida e busca de potenciais.

\section{CONSIDERAÇÕES FINAIS}

Vivemos num momento social em que, de um lado, foram desenvolvidas inúmeras tecnologias de prevenção à gravidez, propiciando maior liberdade sexual às mulheres, e, de outro, se evidenciam o aumento das infecções pelo HIV e a disseminação das doenças sexualmente transmissíveis, exigindo ações concretas de prevenção voltadas ao público feminino. Pode-se observar hoje uma maior freqüência de mulheres grávidas infectadas pelo HIV, e conseqüentemente, um aumento na transmissão do vírus para o bebê durante a gestação, parto ou aleitamento materno.

Uma das formas de identificar precocemente a possibilidade de infecção pelo HIV na mulher grávida é a solicitação do teste, mas este deve ser precedido de aconselhamento. Observou-se que no que diz respeito ao diálogo, entendido como um momento de trocas intersubjetivas, este não ocorre durante a entrega do teste. Os enfermeiros das UBASF deveriam interagir com a clientela privilegiando o vínculo e 0 acolhimento às demandas pessoais.

Por trabalharem na base da produtividade, e não da qualidade, as consultas acontecem num intervalo de tempo muito reduzido. Em uma consulta de pré-natal, onde se espera a realização do aconselhamento para o teste antiHIV, especialmente o pós-teste, deve-se favorecer um período de tempo maior para que se consiga estabelecer efetivamente o diálogo em que a enfermeira e a gestante se relacionem pelo encontro e pela presença.

Portanto, este estudo levanta a necessidade de se repensarem as práticas educativas desenvolvidas pelos enfermeiros nas UBASF, visando a elaborações de abordagens inovadoras e estratégias a serem implantadas pelos profissionais que atuam na assistência integral à saúde da mulher nos serviços de saúde. 
Chama a atenção, ainda, que o cuidado de enfermagem na estratégia de saúde da família se deve voltar para uma abordagem humanista, emancipatória, e não centrada na atenção curativa. Os profissionais devem ter a oportunidade de refletir a sua prática através de

\section{Referências}

1. Tratado de Enfermagem Médico- Cirúrgica. Brunner \& Suddart. $9^{a}$ ed. Rio de Janeiro (RJ): Guanabara Koogan; 2000. v.3.

2. Ministério da Saúde (BR). Boletim Epidemiológico de AIDS, Brasília (DF) 2004 jan/jun. $1: 1$.

3. Ministério da Saúde (BR) Guia de tratamento: recomendações para profilaxia da transmissão vertical do HIV e terapia anti-retroviral em gestante. Brasília (DF); 2004.

4. Connor EM. Reduction of maternal-infant transmission of human immunodeficiency vírus type 1 with zidovudine treatment. J Med 1994; 331(18): 1173-80.

5. Newell ML, Gray G, Bryson YJ. Prevention of mother-to-child transmission of HIV-1. AIDS 1997; 11 (suppl A): S165-S172.

6. Ministério da Saúde (BR). Coordenação Nacional DST/AIDS. Aconselhamento em DST/AIDS para Atenção Básica. Brasília (DF); 2003.

7. Araújo MAL, Bucher JSNF, Bello PY. Eficácia do aconselhamento para doenças sexualmente transmissíveis em unidades de referência da cidade de Fortaleza, CE, Brasil. J Bras Doenças Sexuais Transmissiveis 2004; 16(1): 31-37.

8. Szwarcwald CL. Oportunidades perdidas na detecção precoce do HIV na gestação: resultados do Estudo Sentinela-Parturiente; 2003.

9. Miranda KCL, Vasconcelos KS, Barroso MGT. Aconselhamento em HIV/AIDS: um conceito a partir dos profissionais. Esc Anna Nery Rev Enferm 2003 ago; 7(2): 196-203.

10. Araújo MAL. Avaliação da implementação do aconselhamento e diagnóstico do HIV no pré-natal. [tese de doutorado]. Fortaleza (CE): Universidade Federal do Ceará; 2005.

11. Paterson J,Zderad L. Enfermería humanística. New York(USA): Asociación Nacional de Enfermería; 1988. Publicado originalmente en 1976. processos de educação permanentes, especialmente no que diz respeito ao desenvolvimento das práticas educativas nos serviços de saúde, que devem privilegiar as escolhas pessoais e valorizar o potencial humano.

12. Rogers CR, Rosenberg RL. A pessoa como centro. São Paulo (SP): EPU; 1977.

13. Rosenberg RL. Aconselhamento psicológico centrado na pessoa. EPU. São Paulo (SP); 1987.

14. Araújo MAL, Pagliuca LMF. Análisis de contexto del concepto de ambiente em la Teoria Humanística de Paterson y Zderad. Index de Enfermeria 2005 primavera/verano; 14(48/49): 42-5.

15. Minayo MCS. 0 desafio do conhecimento: pesquisa qualitativa em saúde. São Paulo (SP): HUCITEC; 2004.

16. Trivinos ANS. Introdução à pesquisa em ciências sociais: a pesquisa qualitativa em saúde. São Paulo (SP): Atlas; 1994.

17. Secretaria Municipal de Saúde. Programa de Saúde da Família-PSF. Boletim de Saúde de Fortaleza 2002 jul-set; 6: 3.

18. Ministério da Saúde (BR). Coordenação Nacional DST/AIDS. Aconselhamento em DST/AIDS: diretrizes e procedimentos básicos. Brasília; 2000.

19. Buber M. Eu e tu. $2^{a}$ ed. São Paulo (SP): Cortez \& Moraes; 1979.

\section{Sobre os Autores}

\section{Maria Alix Leite Araújo}

Universidade de Fortaleza - UNIFOR, Fortaleza - CE.

\section{Francisca Lucélia Ribeiro de Farias}

Universidade de Fortaleza - UNIFOR, Fortaleza - CE.

\section{Alanna Virgínia Brito Rodrigues}

Enfermeira. Prefeitura de Caucaia. Caucaia-CE. 